\title{
Coupling Conceptual and Quantitative Problems to Develop Expertise in Introductory Physics Students
}

\author{
Chandralekha Singh \\ Department of Physics and Astronomy, University of Pittsburgh, Pittsburgh, PA, 15260
}

\begin{abstract}
We discuss the effect of administering conceptual and quantitative isomorphic problem pairs (CQIPP) back to back vs. asking students to solve only one of the problems in the CQIPP in introductory physics courses. Students who answered both questions in a CQIPP often performed better on the conceptual questions than those who answered the corresponding conceptual questions only. Although students often took advantage of the quantitative counterpart to answer a conceptual question of a CQIPP correctly, when only given the conceptual question, students seldom tried to convert it into a quantitative question, solve it and then reason about the solution conceptually. Even in individual interviews, when students who were only given conceptual questions had difficulty and the interviewer explicitly encouraged them to convert the conceptual question into the corresponding quantitative problem by choosing appropriate variables, a majority of students were reluctant and preferred to guess the answer to the conceptual question based upon their gut feeling.
\end{abstract}

Keywords: problem solving, introductory physics, conceptual learning, quantitative problems PACS: 01.40Fk,01.40.gb,01.40G-,1.30.Rr

\section{INTRODUCTION}

We investigate whether introductory physics students perform better when they answer a conceptual and quantitative isomorphic problem pair (CQIPP) as a set compared to the case when they answer either the conceptual or the quantitative question of a CQIPP alone. We call the paired problems isomorphic if they require the same physics principle to solve them. Although it is difficult to categorize physics questions as exclusively quantitative or conceptual, CQIPPs had one question that required symbolic or numerical calculation while the other question could be answered by conceptual reasoning alone [1]. We also analyze the performance of students on the CQIPPs from the perspective of "transfer" [2,3].

We developed five CQIPPs in the multiple-choice format with different contexts in mechanics [1]. Below is an example of the first CQIPP (correct answers in italics):

(1) A tugboat pulls a ship of mass $M$ into the harbor with a constant tension force $\vec{F}$ in the horizontal tow cable. Both the tugboat and the ship start from rest. After the ship has been towed a distance $d$ in time $t$, the magnitude of its momentum will be
(a) $F d$
(b) $(1 / 2)(F / M) t^{2}$
(c) $(F / M) t^{2} / d$
(d) $(1 / 2)(F / M) d t^{2}$
(e) $F t$

(2) Two identical tugboats pull other ships as shown below, starting from rest. The Queen Mary is a much more massive ship than the Minnow. Both tugboats pull with the same horizontal force. Neglect other forces. After both tugboats have been pulling for the same amount of time, which one of the following is true about the Queen Mary and the Minnow?

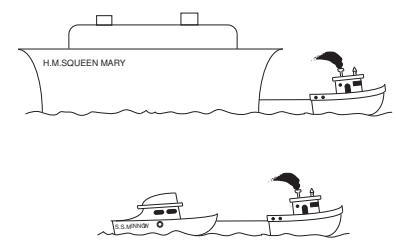

(a) The Queen Mary will have a greater magnitude of momentum.

(b) The Minnow wil have a greater magnitude of momentum.

(c) Both ships will have the same magnitude of momentum.

(d) Both ships will have the same kinetic energy.

(e) The Queen Mary will have a greater kinetic energy.

We made hypotheses $\mathrm{H} 1$ and $\mathrm{H} 2$ as described below:

- H1: Performance on quantitative questions of a CQIPP will be better when both the quantitative and conceptual questions are given than when only the quantitative question is given.

- H2: Performance on conceptual questions of a CQIPP will be better when both the quantitative and conceptual questions are given than when only the conceptual question is given.

Hypothesis H1 is based on the assumption that solving the conceptual question of a CQIPP may encourage 
students to perform a qualitative analysis, streamline students' thinking, make it easier for them to narrow down relevant concepts, and thus help them solve the quantitative problem correctly. Prior studies show that introductory physics students are not systematic in using effective problem solving strategies, and often do not perform a conceptual analysis while solving a quantitative problem. They often use a "plug and chug" approach to solving quantitative problems, which may prevent them from solving the problem correctly. The conceptual questions may provide an opportunity for reflecting upon the quantitative problem and performing a qualitative analysis and planning. This can increase the probability of solving the quantitative problem correctly. We note that since the CQIPPs always had a quantitative question preceding the corresponding conceptual question, hypothesis H1 assumes that students will go back to the quantitative question if they got some insight from the corresponding conceptual question.

Hypothesis $\mathrm{H} 2$ is inspired by prior studies that show that introductory physics students often perform better on quantitative problems compared to conceptual questions on the same topic [4]. Students often treat conceptual questions as guessing tasks [4]. We hypothesized that students who are able to solve the quantitative problem in a CQIPP may use its solution as a hint for answering the conceptual question correctly if they are able to discern the similarity between the two questions. Since quantitative and conceptual questions of a CQIPP were given one after another, we hypothesized that students would likely discern their underlying similarity at least in cases where the contexts were similar. When reasoning without quantitative tools, it may be more difficult to create the correct chain of reasoning if a student is "rusty" about a concept [5]. Equations can provide a pivot point for constructing the reasoning chain. For example, if a student has forgotten whether or not the maximum safe driving speed while making a turn on a curved road depends on the mass of the vehicle, he/she will have great difficulty determining which is correct without using equations to reason. Similarly, a student with evolving expertise who is comfortable reasoning with equations may need to write down Newton's second law explicitly to conclude that the tension in the cable of an elevator accelerating upward is greater than its weight. An expert can use the same law implicitly and conceptually argue that the upward acceleration implies that the tension exceeds the weight without writing down Newton's second law explicitly. Being able to reason conceptually without resorting to quantitative tools in a wide variety of contexts may be a sign of adaptive expertise whereas conceptual reasoning by resorting to quantitative tools may be a sign of evolving expertise [5].

Students in college calculus-based introductory physics courses participated in the study. The questions were asked after instruction in relevant concepts and after students had an opportunity to work on their homework on related topics. When students were given both questions of a CQIPP back to back, the quantitative questions preceded the corresponding conceptual question. However, students were free to go back and forth between them if they wished and could change the answer to the previous question if they acquired additional insight for solving the previous question by answering a latter question. Students who were given both questions of a CQIPP were not told explicitly that the questions given were isomorphic. They were given 2.5 minutes on an average to answer each question. In some of the courses, we discussed the responses individually with several student volunteers.

\section{RESULTS AND DISCUSSION}

Table 1 summarizes the numbers of students who were given both or one question of a CQIPP, and students' average performance. Table 1 also shows the results of a Chi-square test with $p$ values for comparison between cases when both questions of a CQIPP were given vs. only one question was given. Students can make appropriate connections between the questions in a CQIPP only if they have a certain level of expertise that helps them discern the connection between the isomorphic questions. Improved student performance when both questions of a CQIPP were given vs. when only one question was given was taken as one measure of transfer of relevant knowledge from one problem to another.

Table 1 shows that, contrary to our hypothesis H1, student performance on quantitative questions was not significantly different when both quantitative and conceptual questions were given back to back (with the quantitative question preceding the conceptual question) than when only the corresponding quantitative question was given. In some cases, the performance on the conceptual question was better than the performance on the quantitative question (problem pairs (9)-(10)), but students could not leverage their conceptual knowledge for gain on the corresponding quantitative problem. As noted earlier, the two questions in a CQIPP were always given in the same order, although students could go back and forth if they wanted. It is possible that students did not go back to the questions they had already answered, especially due to the time constraint, even if the question that followed provided a hint for it. Future research will evaluate the effect of switching the order of the quantitative and conceptual questions in a CQIPP when both are given.

On the other hand, in support of hypothesis H2, students who worked on both questions of the IPPs involving a conceptual and a quantitative problem performed better on the conceptual questions at least for three of the five IPPs than when they were given only the conceptual 
TABLE 1. For the CQIPP given, the first column lists the problem numbers, the second column gives the percentage of students who chose the correct answer when only one of the questions was given to them, and the third column gives the percentage of students who chose the correct answer when both questions were given. The numbers in parentheses in the second and third columns refer to the number of students who answered the question. The last column for all questions lists the $\mathrm{p}$ value for comparison of student performance between cases when only one of the questions in a CQIPP was given vs. when both questions were given.

\begin{tabular}{|c|c|c|c|}
\hline Problem \# & only one & both & p value \\
\hline 1 & $59(138)$ & $54(289)$ & 0.40 \\
2 & $31(215)$ & $58(289)$ & 0.00 \\
\hline 3 & $34(138)$ & $38(289)$ & 0.45 \\
4 & $23(215)$ & $30(289)$ & 0.07 \\
\hline 5 & $81(138)$ & $76(289)$ & 0.26 \\
6 & $55(215)$ & $80(289)$ & 0.00 \\
\hline 7 & $52(138)$ & $56(289)$ & 0.47 \\
8 & $44(150)$ & $51(289)$ & 0.19 \\
\hline 9 & $49(138)$ & $49(289)$ & 1.00 \\
10 & $53(150)$ & $71(289)$ & 0.00 \\
\hline
\end{tabular}

questions. Table 1 shows that, for three of the IPPs, students often performed better on the conceptual question when both questions were given rather than the corresponding conceptual question alone. The fact that many students took advantage of the quantitative problem to solve the conceptual question points to their evolving expertise. For example, many students who were given both questions (1) and (2) recognized that the final momenta of the ships are independent of their masses under the given conditions by solving the quantitative problem. Written responses and individual discussions suggest that some students who answered the conceptual question (2) correctly were not completely sure about whether the change in momentum in question (1) was given by option (a) or (e). However, since the answer in either case is independent of the mass of the object, these students chose the correct option (c) for question (2). The students who chose the incorrect option (a) for question (1) but the correct option (c) for question (2) often assumed that both ships in question (2) must have traveled the same distance although that is not correct.

In the third CQIPP, question (5) asks students to calculate (numerically) the speed of a person at the bottom of a $5 \mathrm{~m}$ high slide if the person started from rest on the top. Question (6) asks students to compare the speeds of two people with different masses at the bot- tom of the slide, who started from rest at the top of the slide. In individual discussions, several students explicitly noted that the mass cancels out in question (5) so the answer to question (6) cannot depend on mass. In the last CQIPP pairing questions (9) and (10), question (9) asks students to numerically calculate the final speed of a boat moving horizontally when Batman falls vertically into it and comes to rest with respect to the boat. Question (10) asks a conceptual question about the final speed of a cart moving horizontally when rain falls vertically into the cart and comes to rest and also asks students about the physics principle involved in arriving at the solution. Discussions with individual students and students' written work suggest that solving the quantitative question (9) helped many students formulate their solution to question (10). Although some students were not able to solve the quantitative question, e.g., due to algebraic error or not realizing that when considering the conservation of the horizontal component of momentum, Batman's vertical velocity should not be included, it was easier for them to answer the conceptual question after thinking about the quantitative one. Most realized that the boat would slow down after Batman lands in it.

Previous research shows that answering conceptual questions can sometimes be more challenging for students than quantitative ones, if the quantitative problems can be solved algorithmically and students' preparation is sufficient to perform the mathematical manipulations [4]. If a student knows which equations are involved in solving a quantitative problem or how to find the equations, he or she can combine them in any order to solve for the desired variables even without a deep conceptual understanding of relevant concepts. On the contrary, while reasoning without equations, the student must usually proceed in a particular order in the reasoning chain to arrive at the correct conclusion [4]. Therefore, the probability of deviating from the correct reasoning chain increases rapidly as the chain becomes long. We note however that our hypothesis $\mathrm{H} 2$ is not about whether students will perform better on the quantitative or conceptual question of a CQIPP when the two questions are given separately (especially because the wording is not parallel for the quantitative and conceptual questions in a CQIPP). Rather, our hypothesis relates to whether students will recognize the similarity of the quantitative and conceptual questions in a CQIPP, and take advantage of their solution to one question to answer the corresponding paired question. Our finding suggests that students can leverage their quantitative solutions to correctly answer the corresponding conceptual questions, at least in the questions given.

The fact that students often performed better on conceptual questions when they were paired with quantitative questions brings up the following issue. If students could turn the conceptual questions into analogous quan- 
titative problems themselves when only the conceptual questions were given, they may have solved the quantitative problem algorithmically if they were comfortable with the level of mathematics needed, and then reasoned qualitatively about their results to answer the original conceptual question. Almost without exception, students did not do this in the interview situation. One can hypothesize that students have not thought seriously about the fact that a conceptual question can be turned into a quantitative problem, or that a mathematical solution can provide a tool for reasoning conceptually. Without explicit guidance, students may not realize that this conversion route may be more productive than carrying out long conceptual reasoning without mathematical relations. However, we find that students avoided turning conceptual questions into quantitative ones, even when explicitly encouraged to do so. In one-on-one interview situations, when students were only given the conceptual questions, they also tried to guess the answer based upon their gut feeling. More research is required to understand why students are reluctant to transform a conceptual question into a quantitative problem even if the mathematical manipulations required after such a conversion and making correct conceptual inferences are not too difficult for them. One possible explanation for such reluctance is that such a transformation from a conceptual to a quantitative problem is cognitively demanding for a typical introductory physics student and may cause a mental overload [6]. According to Simon's theory of bounded rationality, an individual's rationality in a particular context is constrained by his/her expertise and experience, and an individual will only choose one of the few options consistent with his/her expertise that does not cause a cognitive overload. [7]

In the second CQIPP, problem (3) asks students to calculate the speed of a hoop rolling down a ramp given the various parameters for the hoop and the ramp. Problem (4) asks them to compare the speeds of two different hoops with different masses and radii rolling down the same ramp. For this CQIPP, the quantitative problem was very challenging. Most interviewed students and those who wrote something on their answer sheet did not use conservation of energy correctly and forgot to take into account both the rotational and translational kinetic energies in their analysis. Thus, it is not surprising that there is no significant difference between cases when only one of the questions was given vs. when both were given.

\section{CONCLUSIONS}

While students often took advantage of the quantitative problem to answer the corresponding conceptual question of a CQIPP, those who were only given the corresponding conceptual question did not automatically con- vert it into a quantitative problem as an aid for reasoning correctly. Examination of students' scratch work suggests that they seldom attempted such conversion by choosing appropriate variables. One-on-one discussions suggest that students often used gut feeling to reason about the conceptual questions. This tendency persisted even when the interviewer explicitly encouraged students to convert a conceptual question into a quantitative one. It is possible that converting the conceptual questions to quantitative ones was too cognitively demanding for students and may have caused mental overload.

In this research, isomorphic problems were given back-to-back, and the more quantitative question always preceded the conceptual question in a CQIPP. It is possible that the order in which questions were asked and the proximity of the paired questions in a CQIPP are major factors in whether students will recognize their similarity and transfer relevant knowledge from one problem to another. In future research, one can explore the effect of spacing the questions in a CQIPP and changing the order in which questions are asked on students' ability to benefit from having both questions of a CQIPP.

Presenting quantitative and conceptual isomorphic pairs helped students make conceptual inferences using quantitative tools. Such problem pairs as part of instruction may help students go beyond the "plug and chug" strategy for the quantitative problem solving and may give them an opportunity to reflect upon their solution and develop reasoning and meta-cognitive skills. Solving these paired problems can force students to reflect upon the problem solving process and improve their metacognitive skills. Helping students develop meta-cognitive skills can also improve transfer of relevant knowledge from one problem to another.

\section{ACKNOWLEDGMENTS}

We thank NSF for award DUE-0442087.

\section{REFERENCES}

1. C. Singh, Phys. Rev. ST Phys. Educ. Res. 4, 010105(1-10), (2008).

2. R. Dufresne, J. Mestre, T. Thaden-Koch, W. Gerace and W. Leonard, 155-215, in Transfer of learning from a modern multidisciplinary perspective, J. P. Mestre (Ed.), Greenwich, CT: Information Age Publishing, (2005).

3. D. Ozimek, P. Engelhardt, A. Bennett, N. Rebello, Proc. Phys. Ed. Res. Conf., AIP, 173-176, (2004).

4. L. C. McDermott, Am. J. Phys. 69 (11), 1127-1137, (2001).

5. F. Reif, Am. J. Phys. 63, 17 (1995).

6. J. Sweller, Cognitive Science 12, 257, (1988).

7. Simon, Reason in human affairs, Basil Blackwell, (1983). 